\title{
Necrosectomía endoscópica percutánea en el manejo step-up de walled-off necrosis
}

\author{
José Adi, ${ }^{1}$ Carolina Dutto, ${ }^{1}$ Romina Victoria, ${ }^{1}$ Alfredo Torres, ${ }^{1}$ Belén Bussetti, ${ }^{1}$ \\ Mario Gutiérrez, ${ }^{2}$ Gastón Collaretti ${ }^{2}$ \\ ${ }^{1}$ Servicio de Gastroenterología, Hospital L. C. Lagomaggiore. \\ ${ }^{2}$ Servicio de Cirugía, Hospital L. C. Lagomaggiore. \\ Provincia de Mendoza, Argentina.
}

Acta Gastroenterol Latinoam 2020;50(4):459-462

Recibido: 23/06/2020 / Aceptado: 26/11/2020 / Publicado online: 14/12/2020

\section{Resumen}

El drenaje de colecciones líquidas pancreáticas es de abordaje complejo y desafiante en cuanto a su técnica. En las últimas décadas, los avances endoscópicos han mejorado las tasas de éxito técnico y clinico, con reducción de complicaciones. Presentamos un caso de paciente masculino de 72 años con pancreatitis aguda severa necrotizante tras papilectomía endoscópica por adenoma papilar recidivante, con evolución a walled-off necrosis infectada. Se realiza drenaje percutáneo guiado por tomografía, con mala evolución clinica y persistencia de colecciones, con predominio en cola de páncreas de restos necróticos sólidos y burbujas aéreas. Debido a la localización de la colección y la ausencia de contacto con la pared gástrica, se realiza necrosectomía endoscópica percutánea con colocación de stent metálico esofágico autoexpansible y se consigue la resolución del caso. Esta inusual técnica se plantea con un abordaje step-up de manejo de walled-off necrosis,

Correspondencia: Carolina Dutto

Urquiza 651, San Francisco del Monte, Guaymallén, Mendoza, Argentina Tel.: 2615546244

Correo electrónico: carodutto@gmail.com cuando el drenaje percutáneo es insuficiente y el abordaje endoscópico transgástrico no es posible por las características de la colección. El manejo de walled-off necrosis como complicación tardia de pancreatitis aguda necrotizante requiere un abordaje step-up. La necrosectomía endoscópica alcanza tasas más altas de resolución con menor tasa de complicaciones, tiempo de resolución y recurrencia.

Palabras claves. Walled-off necrosis, necrosectomía endoscópica percutánea.

\section{Percutaneous Endoscopic Necrosectomy in the Step-up Management of Walled-off Necrosis}

\section{Summary}

The drainage of liquid pancreatic collections is complex and technically challenging as for Its technique. In recent decades, endoscopic advances have improved technical and clinical success rates with reduced complications. We present a case of a 72-year-old male patient with severe necrotizing acute pancreatitis after endoscopic papillectomy for recurrent papillary adenoma, with evolution to infected walled-off necrosis. Percutaneous drainage guided by tomography was performed with poor clinical evolution and persistence of collections, predominantly in the tail of the pancreas of solid necrotic remains and air bubbles. Due to the location of the collection and the absence of contact with the gastric wall, percutaneous endoscopic necrosectomy was performed with placement of a self-expanding esophageal metal stent with 
resolution of the case. This unusual technique is proposed in a step-up approach to the management of walled-off necrosis, when the percutaneous drainage is insufficient and the transgastric endoscopic approach is not possible due to the characteristics of the collection. The management of walled-off necrosis as a late complication of acute necrotizing pancreatitis suggests a step-up approach. Endoscopic necrosectomy achieves higher resolution rates with a lower complication rate, resolution time, and cavity recurrence.

Key words. Walled-off necrosis, percutaneous endoscopic necrosectomy.

\section{Introducción}

Las colecciones de fluidos pancreáticos son una complicación frecuente de la pancreatitis. Acerca del 5-15\% de los pacientes desarrollan necrosis del parénquima pancreático o del tejido peripancreático, o de ambos. Esta necrosis puede permanecer estéril o, por el contrario, infectarse, siendo raro que ocurra en la primera semana. ${ }^{1} \mathrm{La}$ presencia de necrosis pancreática infectada hace imperativa la necesidad de drenaje y desbridamiento del tejido afectado. Se ha demostrado en múltiples estudios el beneficio del drenaje endoscópico en comparación con la intervención quirúrgica. No toda colección pancreática posee las características y la localización anatómica adecuada para un abordaje transgástrico, siendo en estos casos una herramienta útil para lograr un punto de acceso percutáneo y el subsecuente desbridamiento endoscópico, lo que permite la necrosectomía directa de la colección. ${ }^{2}$

\section{Caso clínico}

Se presenta el caso de un paciente masculino de 72 años con antecedentes de adenoma papilar con displasia de bajo grado, habiendo sido realizada la primera papilectomía endoscópica en Chile en 2006; posteriormente continuó con controles, pero discontinuos, con una pequeña recidiva en 2009 de fácil resolución endoscópica con histología de adenoma tubular. Ante una nueva recidiva en 2018, se le explican al paciente las posibilidades terapéuticas y se planifica la resección endoscópica. Por asuntos personales del paciente, se posterga el tratamiento hasta 2019 , cuando es recibido en nuestra institución.

Se realiza papilectomía endoscópica y se presenta como complicación una pancreatitis aguda severa necrotizante. A las cuatro semanas, el paciente evoluciona febril, con tomografía computada que evidencia un marcado aumento difuso del volumen y el tamaño del páncreas, asociado a múltiples colecciones heterogéneas, con pared definida amurallada con componente mixto a predominio líquido, imágenes aéreas en su interior localizadas en la región peripancreática, predominando dos colecciones definidas en cuerpo y cola $(69$ x $50 \mathrm{~mm}$ y 77 x $54 \mathrm{~mm}$ ), con extensión a espacio perirrenal, raíz del mesenterio y ambas goteras parietocólicas, sin realce tras la administración de contraste, lo que sugiere necrosis (Figura 1). Debido a la naturaleza predominantemente líquida de las colecciones, se colocan inicialmente dos drenajes percutáneos guiados por tomografía hacia colección de cuerpo y cola, con lavajes diarios con solución fisiológica. Se aísla por cultivo Acinetobacter baumanii. El paciente evoluciona tórpidamente, manteniéndose febril de manera persistente y se agrega falla multiorgánica. En control tomográfico se observa una franca disminución de colección de localización cefálica con persistencia en cola de páncreas en el espacio perirrenal y en espacio gastropancreático, sin contacto con la pared gástrica (Figura 2). Por persistencia de walled-off necrosis en cola de páncreas, ausencia de contacto con pared gástrica o duodenal, y sin contar en la institución con endosonografía sumado a mala respuesta clínica, se decide el abordaje endoscópico transcutáneo con colocación de stent metálico esofágico autoexpandible parcialmente cubierto.

Se realiza procedimiento en quirófano, bajo anestesia, en posición supina. Se avanza alambre guía a través del drenaje percutáneo hacia la cavidad bajo guía fluoroscópica. Posteriormente, se retira el drenaje y se procede a dilatar el tracto con pinza Kocher. Se introduce stent metálico esofágico autoexpandible (120 × 26 × $20 \mathrm{~mm})$. La luz del stent se dilata con balón de $12 \mathrm{~mm}$ en región que corresponde a aponeurosis. Se realiza sutura de la prótesis a la piel y se evidencia salida de material purulento hacia el exterior. Se aborda con un endoscopio a través del stent esofágico hacia la cavidad (Figura 3), observando cavidad con tejido necrótico multicompartimental (Figura 4A). Se realiza necrosectomía por desbridamiento mecánico de residuos necróticos con asa y lavado con solución salina de todos los compartimientos (Figura 4B). En sesión subsiguiente, por evidenciarse presencia de material necrótico subyacente al stent, se retira hacia el exterior $3 \mathrm{~cm}$, continuando con necrosectomía. Se realizan sesiones cada 48 horas con un total de cinco, hasta lograr visualizar ausencia de material necrótico, con paredes constituidas por tejido de granulación, indicando vitalidad del mismo (Video) (Figura 4C). Todos los procedimientos fueron realizados por un solo operador. 
Figura 1. STC de abdomen con colecciones pancreáticas heterogéneas con aire en su interiors

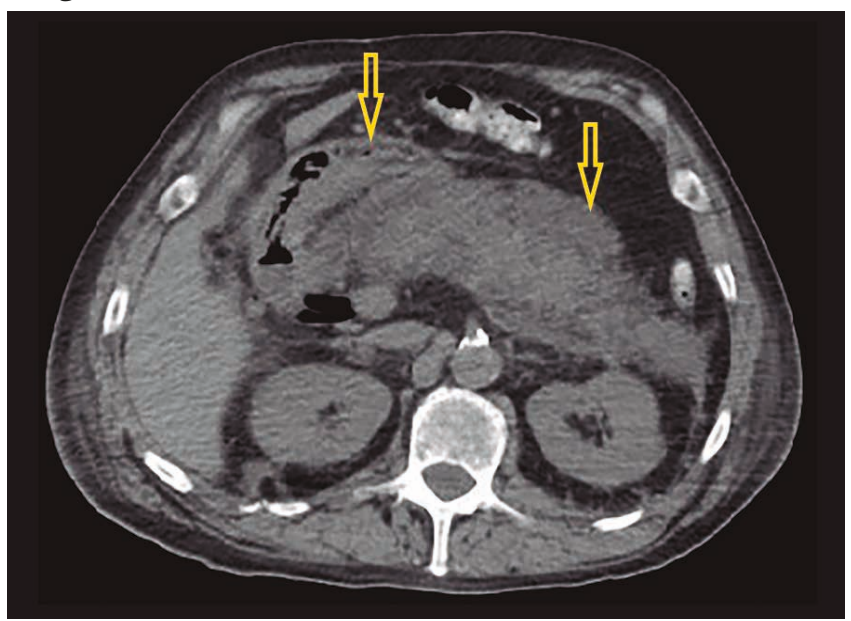

Figura 2. TC axial de abdomen. Se observa persistencia de colección heterogénea en cola de páncreas luego de drenaje percutáneos

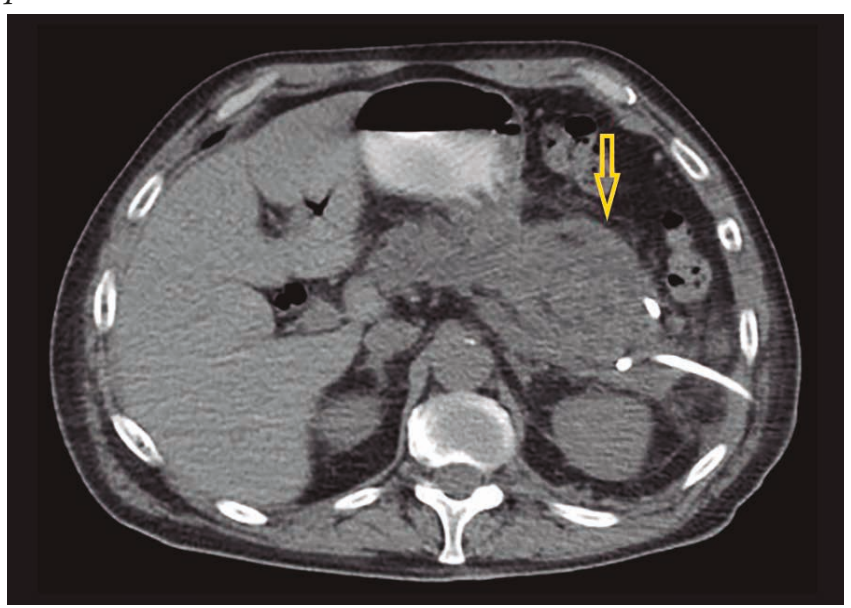

Figura 3. Necrosectomía endoscópica a través de stent metálico esofágico transcutáneo

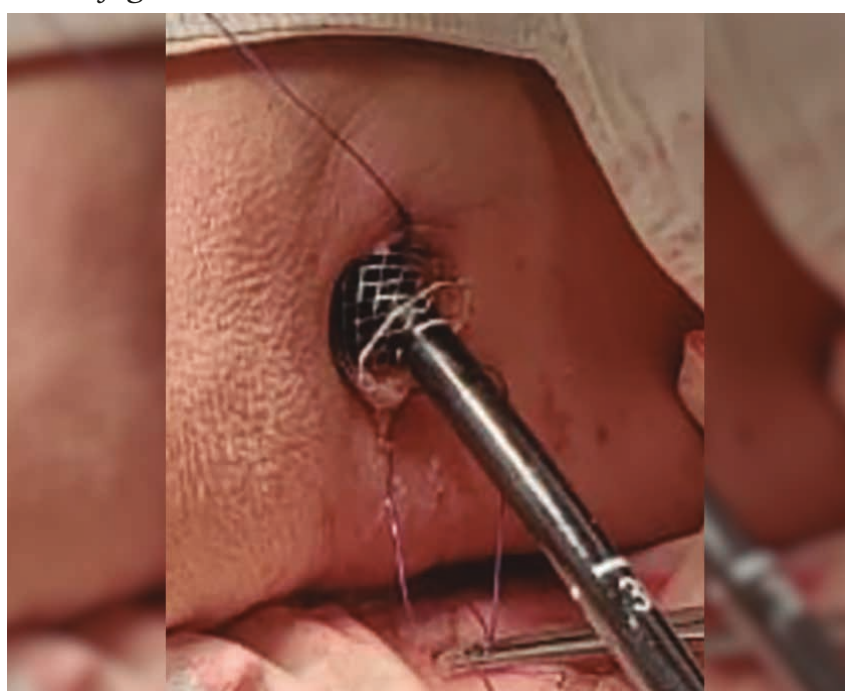

Figura 4. Necrosectomía endoscópica transcutánea. A) Cavidad multicompartimental con tejido necrótico. B) Necrosectomía con asa. C) Paredes de cavidad con tejido de granulación
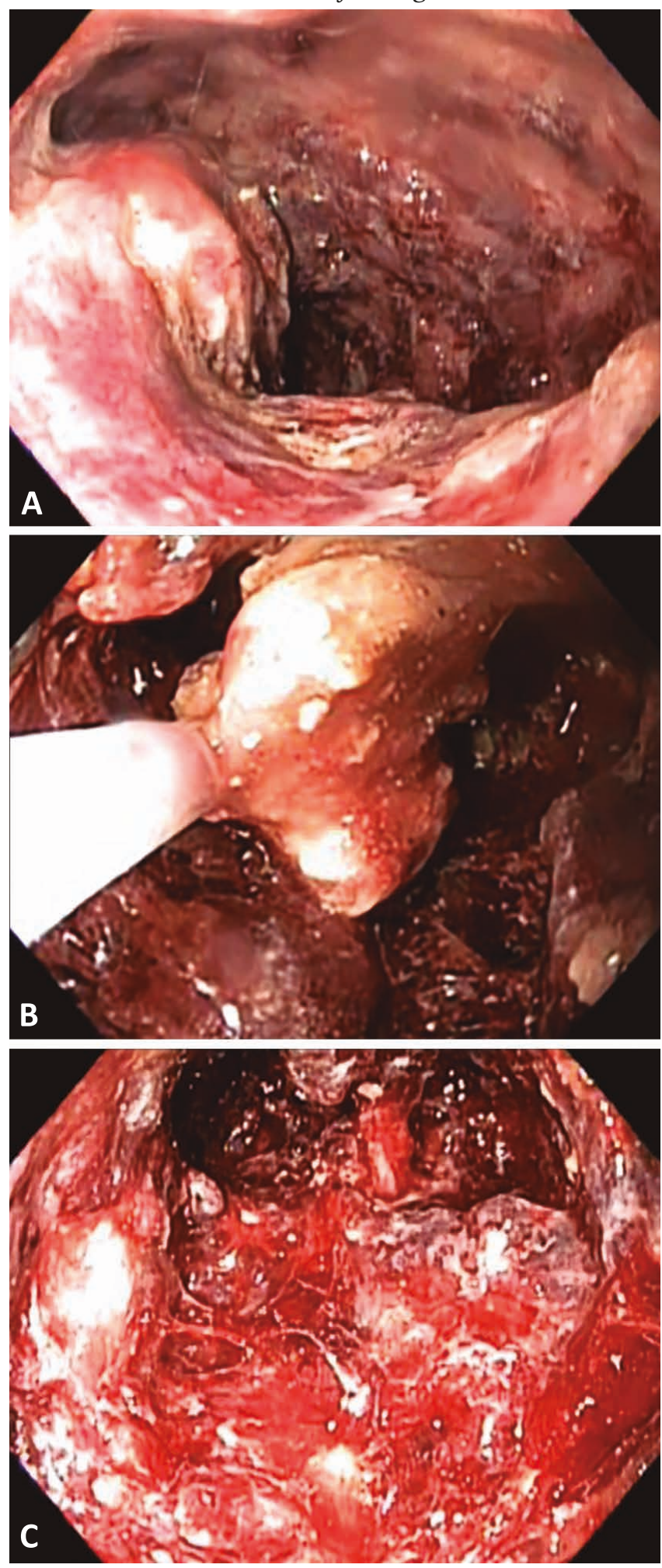

Video. Puede visualizarse el video en: https://www.youtube. com/watch? $v=q T f 6 d J c p m H I$ 
El paciente evoluciona favorablemente con mejoría clínica y tomográfica. Se retira el stent luego de la última sesión y se coloca un drenaje, permaneciendo un escaso débito por diez días, con niveles de amilasa normal, dejándose curar el tracto fistuloso por intención secundaria (Figura 5). El paciente continuó dos semanas internado para control evolutivo, con posterior alta hospitalaria.

Figura 5. TC corte axial. Se observa resolución de colecciones pancreáticas, con drenaje percutáneo multipropósito con extremo distal hacia cola de páncreas

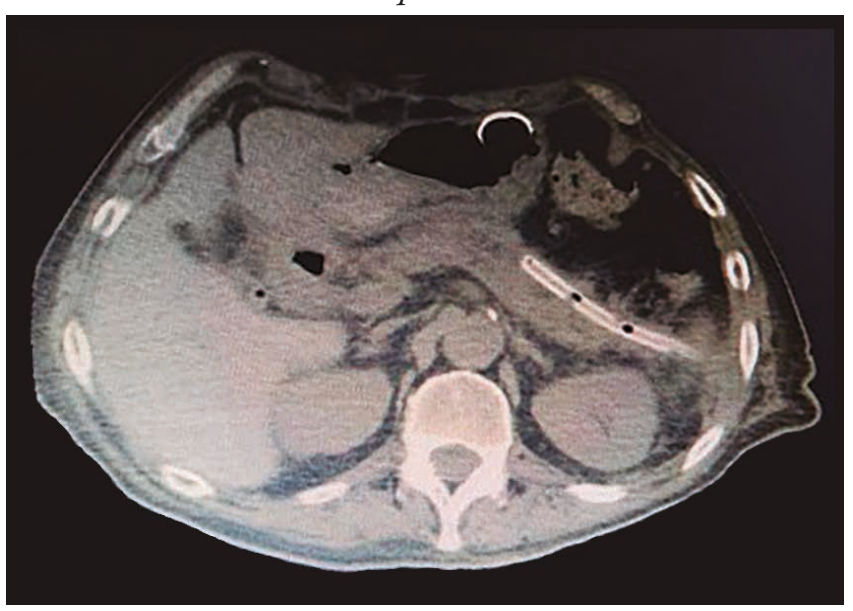

\section{Discusión}

Aproximadamente el $20 \%$ de los pacientes con pancreatitis aguda desarrollan necrosis pancreática; de la totalidad de los pacientes, acerca del 30\% evolucionan a pancreatitis necrotizante infectada.3 La pancreatitis aguda poscolangiopancreatografía retrógrada endoscópica es una complicación con una incidencia que ronda el 3,5-9,7\%, donde el $85 \%$ resuelven sin complicaciones, pero el $15 \%$ eventualmente presenta necrosis que requiere intervención invasiva. ${ }^{4,5} \mathrm{El}$ abordaje step-up de necrosis pancreática infectada es ampliamente difundido y aceptado en el mundo. En las últimas dos décadas, debido a la mejoría de herramientas y técnicas no invasivas, se ha preferido el abordaje endoscópico por haber demostrado mejores tasas de éxito clínico, menor morbimortalidad y menores costos en comparación con el desbridamiento quirúrgico. ${ }^{6,7}$ Ciertas características de la colección, ya sean múltiples cavidades, tamaño y porcentaje de necrosis, así como la localización, que contraindican un drenaje transgástrico, pueden plantear un desafío técnico en cuanto al acceso a través del tracto gastrointestinal hacia la colección. ${ }^{2}$ En estos casos, el drenaje percutáneo endoscópico con colocación de stent metálico, técnica descrita y publicada inicialmente por el Dr. Claudio Navarrete, ${ }^{8}$ plantea una opción válida con alta tasa de éxito terapéutico y clínico, proporcionan- do una ruta confiable a través de stent metálico transcutáneo para realizar necrosectomía repetida retroperitoneal o transperitoneal, de acuerdo con la localización y la extensión de tejido necrótico, con posibilidad de realizarlo sin anestesia general, con sedación consciente y "bedside"., 10

\section{Conclusión}

El manejo de walled-off necrosis es complejo y el step-up depende de las características de la cavidad y de los recursos médicos disponibles. El desbridamiento endoscópico presenta mejores tasas de éxito y menores complicaciones. La decisión de abordaje dependerá de las características del paciente, de la colección y de la experiencia del centro médico, siendo fundamentales la discusión y el manejo multidisciplinario.

\section{Referencias}

1. Banks P, Bollen T, Dervenis C, Gooszen H, Johnson C, Sarr M, Tsiotos G, Swaroop Vege S. Acute Pancreatitis Classification Working Group. Classification of acute pancreatitis - 2012: revision of the Atlanta classification and definitions by international consensus. Gut 2013; 62: 102-111.

2. Saumoy M, Kumta NA, Tyberg A, Brown E, Lieberman MD, Eachempati SR, Winokur RS, Gaidhane M, Sharaiha RZ, Kahaleh M. Transcutaneous endoscopic necrosectomy for walled-off pancreatic necrosis in the paracolic gutter. J Clin Gastroenterol 2018; 52 (5): 458-463.

3. Jha AK, Goenka MK, Kumar R, Suchismita A, Endotherapy for pancreatic necrosis: An update. JGH Open: An open access journal of gastroenterology and hepatology 2019: 80-88.

4. Dumonceau JM, Kapral C, Aabakken L, Papanikolaou IS, Tringali A, Vanbiervliet G, Beyna T, Dinis-Ribeiro M, Hritz I, Mariani A, Paspatis G, Radaelli F, Lakhtakia S, Veitch AM, Van Hooft JE. ERCP-related adverse events: European Society of Gastrointestinal Endoscopy (ESGE) Guideline. Endoscopy 2020; 52 (02): 127-149.

5. Arvanitakis M, Dumonceau JM, Albert J, Badaoui A, Bali MA, Barthet M, Besselink M, Deviere J, Oliveira Ferreira A, Gyökeres T, Hritz I, Hucl T, Milashka M, Papanikolaou I, Poley JW, Seewald S, Vanbiervliet G, Van Lienden K, Van Santvoort H, Voermans R, Delhaye M, Van Hooft J. Endoscopic management of acute necrotizing pancreatitis: European Society of Gastrointestinal Endoscopy (ESGE) evidence-based multidisciplinary guidelines. Endoscopy 2018; 50: 524-546.

6. Binmoeller KF, Nett A. The evolution of endoscopic cystgastrostomy. Gastrointest Endoscopy Clin N Am 2018; 28: 143-156.

7. Van Brunschot $S$, et al. Endoscopic or surgical step-up approach for infected necrotising pancreatitis: a multicentre randomised trial. The Lancet 2017; 3 .

8. Navarrete C, Castillo C, Caracci M, et al. Wide percutaneous access to pancreatic necrosis with self-expandable stent: new application (con video). Gastrointest Endosc 2011; 73: 609.

9. Tingarali A, Vadalà di Prampero S, Costamagna G. Endoscopic necrosectomy of walled-off pancreatic necrosis by large-bore percutaneus metal stent: a new opportunity? Endoscopy International Open 2018; 6: E274-E278.

10. Ke L, Mao W, Zhou J, Ye B. Stent-Assisted Percutaneous Endoscopic Necrosectomy for Infected Pancreatic Necrosis: Technical Report and a Pilot Study. World J Surg. 2019; 43 (4): 1121-1128. 